\title{
Development of the single-mode fiber integral field unit for the RHEA Spectrograph
}

Adam D. Rains, Michael J. Ireland, Nemanja Jovanovic, Joao Bento, Tobias Feger, et al.

Adam D. Rains, Michael J. Ireland, Nemanja Jovanovic, Joao Bento, Tobias Feger, Julien Lozi, Christian Schwab, David W. Coutts, Olivier Guyon, Alexander Arriola, Simon Gross, Jonathan E. Harris, "Development of the single-mode fiber integral field unit for the RHEA Spectrograph," Proc. SPIE 10702, Ground-based and Airborne Instrumentation for Astronomy VII, 107025J (6 July 2018); doi: 10.1117/12.2314336

Event: SPIE Astronomical Telescopes + Instrumentation, 2018, Austin, Texas, United States 


\title{
Development of the single-mode fiber integral field unit for the RHEA Spectrograph
}

Adam D. Rains ${ }^{\mathrm{a}}$, Michael J. Ireland ${ }^{\mathrm{a}}$, Nemanja Jovanovic ${ }^{\mathrm{b}}$, Joao Bento ${ }^{\mathrm{a}}$, Tobias Feger ${ }^{\mathrm{d}}$, Julien Lozic $^{\mathrm{c}}$, Christian Schwab ${ }^{\mathrm{d}, \mathrm{e}}$, David W. Coutts ${ }^{\mathrm{d}}$, Olivier Guyon ${ }^{\mathrm{c}, \mathrm{f}, \mathrm{g}, \mathrm{h}}$, Alexander Arriola ${ }^{\mathrm{d}, \mathrm{i}}$, Simon Gross $^{\mathrm{d}, \mathrm{i}}$, and Jonathan E. Harris ${ }^{\mathrm{j}}$

\author{
aResearch School of Astronomy and Astrophysics, Australian National University, Canberra, \\ ACT 2611, Australia \\ ${ }^{\mathrm{b}}$ Caltech Optical Observatory, Department of Astronomy, California Institute of Technology, \\ 1200 E. California Blvd. Pasadena, CA, 91125 \\ 'Subaru Telescope, National Astronomical Observatory of Japan, National Institutes of \\ Natural Sciences (NINS), 650 North A'Ohoku Place, Hilo, HI, 96720, U.S.A. \\ ${ }^{\mathrm{d}}$ Department of Physics and Astronomy, Macquarie University, NSW 2109, Australia \\ eAustralian Astronomical Observatory, 105 Delhi Rd, North Ryde NSW 2113, Australia

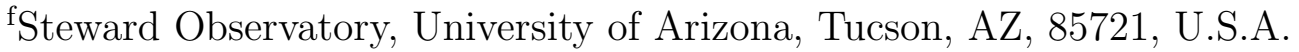 \\ ${ }^{\mathrm{g}}$ College of Optical Sciences, University of Arizona, Tucson, AZ 85721, U.S.A. \\ h Astrobiology Center of NINS, 2-21-1, Osawa, Mitaka, Tokyo, 181-8588, Japan \\ ${ }^{\mathrm{i}}$ Centre for Ultrahigh bandwidth Devices for Optical Systems (CUDOS) \\ jDepartment of Physics, University of Bath, Bath, BA2 7AY, UK
}

\begin{abstract}
RHEA is a single-mode échelle spectrograph designed to be a replicable and cost effective method of undertaking precision radial velocity measurements. The instrument has a novel fiber feed with an integral field unit injecting into a grid of single-mode fibers reformatted to form a pseudo-slit, increasing throughput and enabling highspatial resolution observations when operating behind Subaru and the SCExAO adaptive optics system. The past 18 months have seen a replacement cable constructed for the instrument to address modal noise caused by closely packed fibers with similar path lengths. Here we detail the cable fabrication procedure, design improvements, increased precision in meeting the required sub-micron optical tolerances, throughput gains, and known remaining issues.
\end{abstract}

Keywords: spectrograph, radial velocity, optical fibers, fiber injection, diffraction-limited spectrograph, high resolution, integral field unit

\section{INTRODUCTION}

The Replicable High-resolution Exoplanet and Asteroseismology Spectrograph, RHEA for short, is a compact, single-mode fiber (SMF) fed, échelle spectrograph, conceived and built with the goal of creating a replicable and cost-effective device with which to undertake precision radial velocity (RV) measurements. ${ }^{1-3}$ To increase throughput, RHEA is equipped with a unique optical fiber feed, employing a diffraction-limited miniature integral field unit (IFU) to inject light into a 2-dimensional grid of SMF which, when reformatted into an array, forms the pseudo-slit of the spectrograph.

The primary objective of the RHEA program was long-term precision RV monitoring from a distributed network of automated telescopes, capitalizing on the instrument's inexpensive and technically mature design, as well as the dedicated nature of the network. In such a setup, RHEA could establish large observational

Further author information: (Send correspondence to A.Rains)

A.Rains: E-mail: adam.rains@anu.edu.au

Ground-based and Airborne Instrumentation for Astronomy VII, edited by Christopher J. Evans, Luc Simard, Hideki Takami, Proc. of SPIE Vol. 10702, 107025J · @ 2018 SPIE · CCC code: 0277-786X/18/\$18 · doi: 10.1117/12.2314336 
time baselines on bright exoplanet host stars, eventually enabling for the disentanglement of exoplanet and asteroseismic signals. For this observational program, using only small telescopes (diameters $<50 \mathrm{~cm}$ ), and minimal adaptive optics (AO) in the form of tip-tilt correction, RHEA's IFU and fiber cable exists to offset the principle disadvantage of using SMFs by increasing throughput, particularly in the presence of atmospheric turbulence, through the small, but modal noise free, fibers.

However, when RHEA is placed behind an extreme adaptive optics system on an $8 \mathrm{~m}$ class telescope, the IFU becomes capable of spatially resolving targets at the 11 milli-arcsecond level at high-spectral resolution, opening up a wide (and entirely different) array of science cases to the instrument. These include, but are not limited to, velocity maps of spatially resolved stellar photospheres and outflows, scattered light from protoplanetary disks, and the direct detection of accreting protoplanets.

The $8.2 \mathrm{~m}$ Subaru Telescope and the Subaru Coronographic Extreme Adaptive Optics (SCExAO) system ${ }^{4}$ atop Mauna Kea in Hawaii make this possible. The Subaru copy of RHEA, known as RHEA@Subaru, ${ }^{5}$ is a prototype IFU behind an extreme AO system, designed for bright resolved star science and as a prototype for future high dispersion coronagraphy. It was commissioned using SCExAO engineering time in early 2016, largely in "piggy-back" mode using wavelengths neither required for wavefront sensing or infrared instruments. RHEA@Subau is the first optical (R-band) IFU behind an extreme AO system, with parameters as per Table 1. RHEA's small IFU does not equip it to be a discovery or survey machine. Rather, it provides excellent follow-up of isolated targets, or objects barely resolved with only the largest of modern telescopes.

\begin{tabular}{l|l} 
Table 1. Design specifications of the RHEA@Subaru spectrograph and IFU. \\
\hline Spatial Resolution & 11 milli-arcsec per lenslet \\
Spectral Resolution & R $\sim 60,000$ \\
Total Field of View & $\sim 4$ arcsec \\
Instantaneous Field of View & 40 milli-arcsec \\
IFU Elements & 9 (with dithering capability) \\
Wavelength Range & $590-810 \mathrm{~nm}$ \\
\hline
\end{tabular}

As per the discussion in 5, the original cable for RHEA@Subaru was found to be flawed in that it was afflicted with modal noise due to the closely packed nature the near identical length SMFs. This has since been remedied with a replacement cable, the third fiber cable fabricated for the RHEA program, and the most advanced in terms of fiber alignment procedure and structural stability. Despite this, the measured system throughput at Subaru is currently significantly lower than the predicted value. As such, the cause of this discrepancy, as well as advancements in the cable manufacturing and characterization techniques, are the primary motivation for the following proceedings.

In this paper we begin with a short overview of the spectrograph itself in Section 2. Section 3 covers the fiber cable, going into detail on each component and the fabrication process of both the original cable and its replacement. Characterization of the fiber cable and instrument in terms of fiber alignment and system (component-wise) throughput take place in Sections 4 and 5 respectively. The future of the instrument is discussed in Section 6, before finally summarizing this work in Section 7.

\section{INSTRUMENT DESCRIPTION}

The RHEA@Subaru spectrograph is almost identical to the instrument described in 1-3, receiving only minor modifications for the Subaru version. Operating at a resolution of 75,000 to 50,000 and over a wavelength range of 590-810 $\mathrm{nm}$, RHEA is a prism cross-dispersed near-littrow échelle spectrograph, with a $\sim 13 \mathrm{~mm} 1 / e^{2}$ beam width. Thermal control maintains the spectrograph optics at $<5 \mathrm{mK}$ and $<10 \mathrm{mK}$ precisions in lab and telescope environments respectively. The remaining instrument parameters for RHEA@Subaru are as described in Table 2, and the departures from the design in previous publications as follow:

- Multi-fiber input at the spectrograph slit,

- Installed, but not operational grating vacuum chamber, 
- Two cross-dispersion prims to allow for a larger slit width and longer wavelengths,

- Different grating angle to allow for said longer wavelengths.

Table 2. Summary of RHEA properties ${ }^{3}$

\begin{tabular}{|l|c|}
\hline \multicolumn{1}{|c|}{ Parameter } & Value \\
\hline Spectrograph working aperture & $\mathrm{f} / 15.4$ \\
\hline Fiber link & SMF $630 \mathrm{HP}, 12 \mathrm{~m}, 0.13 \mathrm{NA}$ \\
\hline Beam Size & $590-810 \mathrm{~mm}\left(1 / \mathrm{e}^{2}\right)$ \\
\hline Wavelength range & $\lambda / \Delta \lambda \approx 60,000(700 \mathrm{~nm})$ \\
\hline Resolving power & 45 orders \\
\hline Number of orders & $\sim 2.5$ pix per FWHM \\
\hline Pixel sampling & Infinity-corrected tube lens, $f=200 \mathrm{~mm}(f / 6.7)$ \\
\hline Collimator/camera & N-SF5 prism, $40^{\circ}$ apex angle, $\times 2($ in series $)$ \\
\hline Cross-disperser & N-BK7 $3^{\circ} 53^{\prime}$ apex angle \\
\hline Vacuum window & R2, 31.6 grooves mm ${ }^{-1}, 50 \times 25 \mathrm{~mm}$ ruled area \\
\hline Échelle grating & Sony ICX694AL Exview CCD \\
\hline CCD Detector & $2750 \times 2200,4.54 \mu m, 77 \%$ QE $(580 \mathrm{~nm})$, Grade 1 \\
\hline Cooled detector package & Starlight Xpress Trius-SX694 \\
\hline
\end{tabular}

\section{FIBER CABLE}

As previously described, the RHEA@Subaru's fiber cable takes a 3x3 grid of SMF as input, and places them in a linear (v-groove) array at the spectrograph slit. A tenth fiber introduced at the slit end (separated by a one fiber gap) enables for calibration and simultaneous RV monitoring when illuminated by white-light or neon references sources respectively. The cable described in 5 , the first such cable fabricated for the RHEA program, was in many respects a prototype, with the current RHEA@Subaru cable boasting a number of design improvements as detailed in Table 3. The most critical of these exists to address the modal noise observed in the original cable, a problem resolved through the insertion of path length differences between adjacent fibers at the slit end. Both ends of the completed cable are shown in Figure 1, with more information on their design requirements and assembly presented in Sections 3.1 and 3.2.

Table 3. Comparison of fiber cables fabricated.

\begin{tabular}{|c|c|c|c|c|}
\hline Cable & Fiber & \# Fibers & Length & Features \& Improvements \\
\hline Original & $630 \mathrm{HP}$ & $9+1$ & $\sim 12 \mathrm{~m}$ & $\begin{array}{l}\text { Far field active optical alignment. } \\
\text { 3-axis directional alignment }(\mathrm{X}, \mathrm{Y}, \mathrm{Z}) \text {. } \\
\text { Stainless steel ferrules. } \\
\text { Reference fiber for simultaneous calibration. } \\
8 \text { milli-arcsec on-sky resolution per lenslet. }\end{array}$ \\
\hline Replacement & $630 \mathrm{HP}$ & $9+1$ & $\sim 12 \mathrm{~m}$ & $\begin{array}{l}\text { Near \& far field active optical alignment. } \\
\text { 3-axis directional alignment }(\mathrm{X}, \mathrm{Y}, \mathrm{Z}) \text {. } \\
\text { 3-axis angular alignment }\left(\theta_{X}, \theta_{Y}, \theta_{Z}\right) \text {. } \\
\text { Glass ferrules with tight inner diameter }(\oslash) \text {. } \\
\text { Reference fiber for simultaneous calibration. } \\
11 \text { milli-arcsec on-sky resolution per lenslet. } \\
\text { Improved alignment \& gluing procedures. } \\
\text { Reduced cross-talk on detector. } \\
\text { Improved cable strength. } \\
\text { Glass mask to locate \& support ferrules. } \\
\text { Anti-reflection coatings on all optics. }\end{array}$ \\
\hline
\end{tabular}

\subsection{Integral Field Unit}

The IFU itself is composed of an OKOTech microlens array (APO-Q-P1000-F4,64 (633), fused silica, 2.12mm radius of curvature, $4.64 \mathrm{~mm}$ focal length), and a pair of Thorlabs unmounted anamorphic prisms (PS870), 

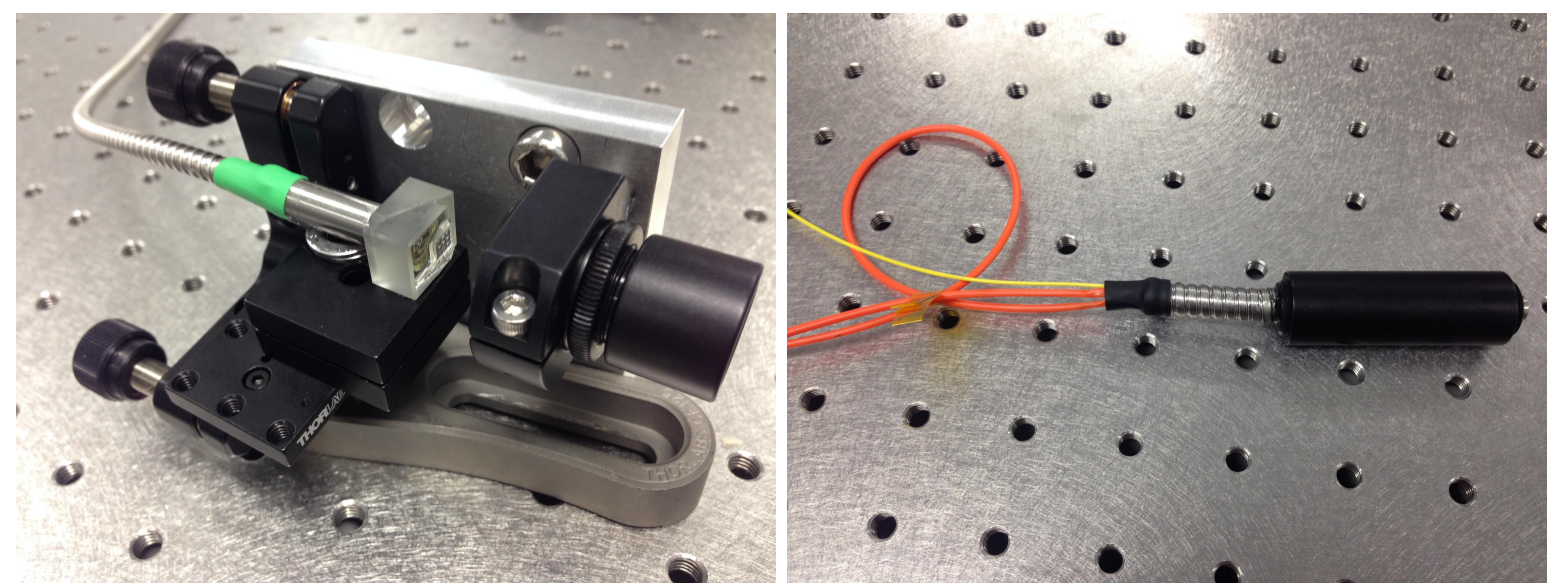

Figure 1. Left: Completed IFU for replacement RHEA@Subaru cable prior to shipment to Hawaii. The IFU is mounted on the SCExAO alignment optics with $\theta_{Y}, \theta_{X}$ and focus adjustments possible. Right: Sealed RHEA@Subaru cable and completed slit-optics. The orange cables contain the 9 science fibers (the loop a result of fiber path length differences), and the yellow cable contains the simultaneous reference fiber.

bonded together with index matching NOA 61 UV curing glue. The use of sliding prisms aided in achieving the designed glass separation between the microlens array and surface to be used for fiber gluing, as contrasted with the more complex and expensive process of glass machining.

By changing the designed microlens-source separation, the on-sky resolution of each lenslet can be selected, influencing the overall coupling in the process. The original cable was fabricated for an on-sky resolution of 8 milli-arcsec per lenslet, but by reducing this distance and opting instead for higher throughput (by nearly a factor of 2), its replacement has a resolution of 11 milli-arcsec per lenslet and needs only to consider a different dither pattern.

Another departure from the original cable was to use glass, rather than stainless steel, ferrules for rigidity on the fiber tips. This offered two main improvements on the original design:

1. UV-curing glue could be cured through the ferrules to bond them to the fibers, removing the constraints placed on gluing by more viscous, and quicker setting, epoxy. By having a defined setting time once mixed, the epoxy made it difficult to have a consistent gluing procedure for each fiber, resulting in bubbles forming in the glue, and rendering the fiber polishing procedure more difficult,

2. The glass ferrules were specifically selected to have much tighter inner diameters (see Figure 2, thus requiring less glue (plus a correspondingly smaller opportunity for bubbles), and reducing much of the potential for the fiber to become offset (i.e. non-concentric with respect to the ferrule).

Polishing of the ferrule-clad fiber tips was done using incrementally finer grained polishing paper ( $5 \mu \mathrm{m}$ to $0.1 \mu \mathrm{m})$, with the refractive index matching nature of the UV-curing glue able to account for minor imperfections in an otherwise unchipped and polishing-debris-free core.

In an effort to provide additional stability to bonded fibers, which received no extra support in previous cables beyond stainless-steel ferrules and their bond directly to the glass prism, a glass mask (Figure 2) was incorporated into the design. The mask, bonded to the prism-unit prior to optical fiber alignment, served to provide additional lateral strength to the fibers, preventing them from being jostled out of position once being glued in place, and restricting potential fiber movement during glue curing. Relative to the $410 \mu \mathrm{m}$ diameter fibers, the $500 \mu \mathrm{m}$ holes machined into the mask provide enough space to maneuverer the ferrule-clad fibers during positioning, whilst still being suitably tight to provide strength once the cavity has been filled with glue and cured.

A three camera active optical alignment procedure was used in the fabrication of the IFU, as shown in Figure 3. A six-axis stage $\left(\mathrm{X}, \mathrm{Y}, \mathrm{Z}, \theta_{X}, \theta_{Y}, \theta_{Z}\right.$, ) was used for fiber positioning and alignment, guided by an overhead 

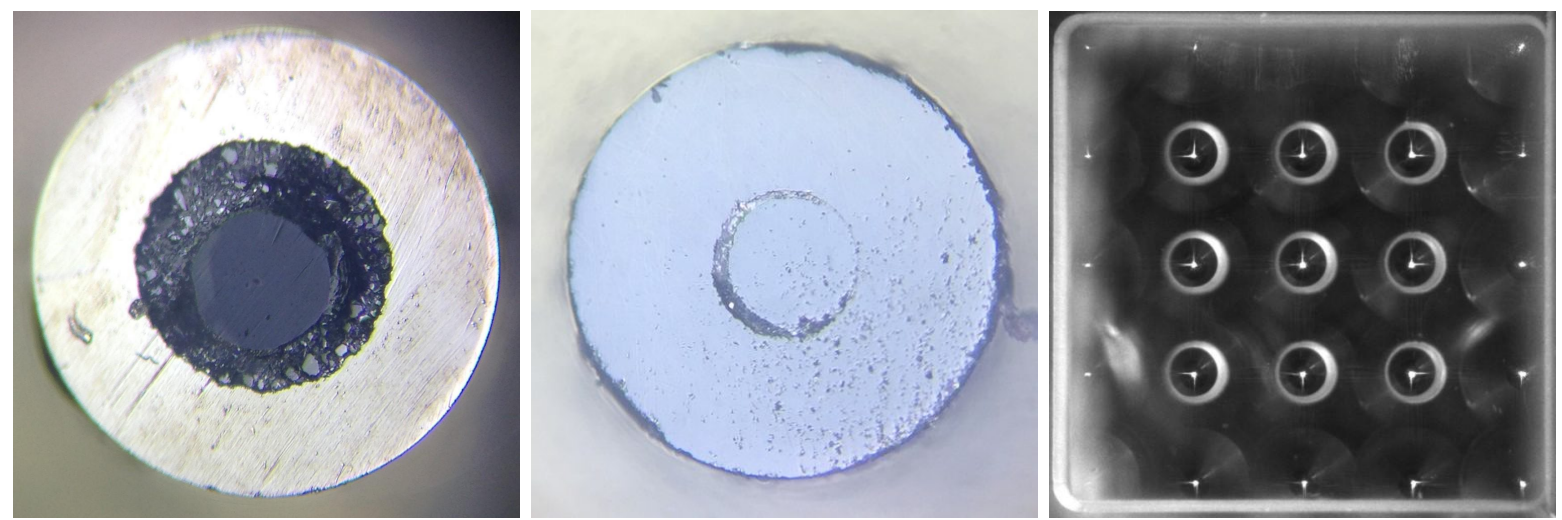

Figure 2. Comparison of polished ferrules and adhesives, and glass fiber locating mask. Left: Metal and epoxy $[O D=$ $400 \mu \mathrm{m}$, fiber cladding $\oslash=125 \mu \mathrm{m} \pm 1.5 \mu \mathrm{m}]$ Center: Glass and UV-curing glue $[O D=410 \mu \mathrm{m}, I D=135 \mu \mathrm{m}$, fiber cladding $\oslash=125 \mu \mathrm{m} \pm 1.5 \mu \mathrm{m}$ ] Right: $1.15 \mathrm{~mm}$ pitch grid, $0.5 \mathrm{~mm}$ hole diameter glass mask during alignment from the perspective of the imaging camera. Note the IFU microlens array bringing light to focus at the centre of each hole.

microscope, and two cameras for imaging the near and far fields of the back-illuminated fibers. The first fiber glued served as a continually-illuminated fiducial, with all subsequent fibers having their far fields (i.e. the common pupil plane) aligned with respect to it. Following all 9 fibers being bonded, additional UV-curing glue was added for strength, and a rigid metal "neck" epoxied into place to further protect the now complete IFU.

\subsection{Slit Optics}

As shown in Figure 1, the slit end of the cable forms a sealed unit, fed by the reference fiber and science fibers of two different path lengths, each contained in their own length of furcation tubing. As mentioned in 5 and earlier in these proceedings, an unexpected flaw was identified in the original RHEA@Subaru cable in the form of "modal noise". The closely packed fibers in the v-groove had almost identical path lengths, resulting in their electric fields overlapping on the detector. For small path length differences $\left(<\lambda^{2} / \delta \lambda\right)$, either constructive or destructive interference occurred on each pixel, an effect sensitive to the injection conditions, cable temperature and temperature gradients. To combat this, the replacement cable was fabricated with path length differences between neighboring fibers in the slit, achieved in practice by alternating the lengths in the pattern long-shortlong-short. This resolves the issue, at the expense of a slightly more complex design.

The slit of RHEA's cable consists of each science fiber, along with the simultaneous reference fiber, being arranged in a v-groove - a piece of glass or silicon with parallel ' $\mathrm{V}$ ' shaped channels locate each fiber relative to its neighbours. Given the cable for RHEA@Subaru was to receive light from a resolved source (i.e. each IFU element, and thus fiber, will observe a different part of the target), consideration had to be given to the ordering of each fiber in the array to reduce unnecessary crosstalk for non-adjacent IFU elements on the detector. Whilst not possible to unwrap a 2D grid to a 1D array while maintaining all fiber proximities, using a winding continuous "snake" pattern is optimal, rather than discontinuous strategies such as row by row, or even arbitrary selection. Following the unwrapped fibers in the v-groove, the continuous reference fiber was preceded by a one fiber gap to minimize crosstalk. The v-groove was then sealed with UV-curing glue, mounted, and subsequently polished using the same process as the ferrule-clad fibers.

A schematic view of the slit optics is shown in Figure 4, showing the optics for injection into RHEA itself. The polished v-groove is aligned and bonded to a second microlens array to bring the light from the fibers individually to focus, and the two larger lenses serving to demagnify and bring the images of the microlens array and fiber cores to focus on the CCD and grating respectively, thus completing RHEA's pseudo-slit. 

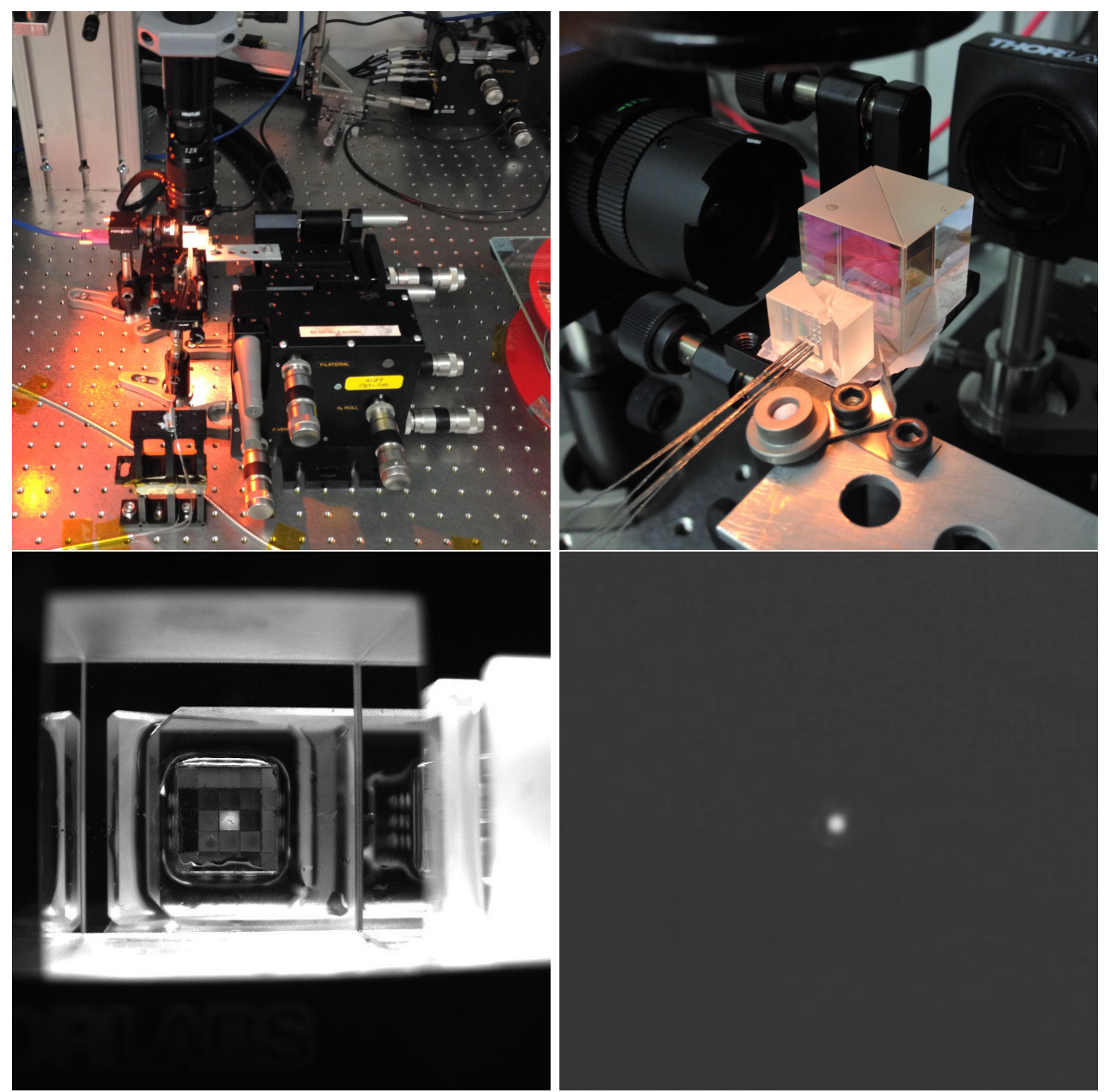

Figure 3. IFU fiber gluing setup. Top-Left: IFU gluing bench setup, consisting of a 6-axis stage, overhead microscope, two imaging cameras, and IFU/beam splitter platform. Top-Right: Two camera alignment setup with IFU, beam splitter and fiber clamp. Bottom-Left: Corresponding near field camera view (i.e. the surface of the microlens array) for the same illuminated central fiber. Bottom-Right: Far field camera view (i.e the common fiber pupil plane) for the illuminated central fiber.

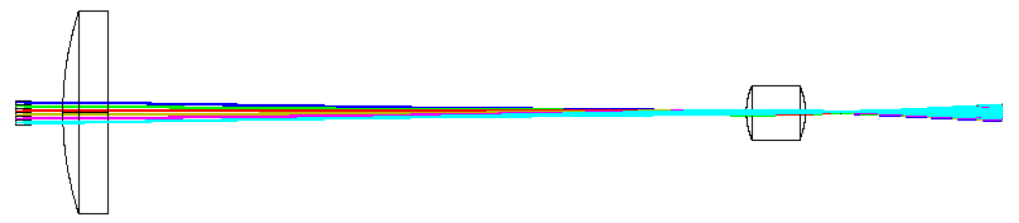

Figure 4. Zemax layout of slit optics for RHEA@Subaru (12 channel OzOptics v-groove, SUSS 18-00030 microlens array, Thorlabs LA1304-B, and N414TM-B), optimized for input F-ratio of 15.4, pixel size of $4.54 \mu \mathrm{m} \mathrm{px}^{-1}$, and minimum order spacing of $43 \mathrm{px}$.

\section{FIBER ALIGNMENT CHARACTERIZATION}

Completing the IFU manufacture and fiber alignment to arbitrary precision should result in the light from all fibers coming to focus and overlapping at their common pupil plane when back-illuminated. However, the challenging procedure resulted in offsets slightly outside our design tolerances, and can be determined through the comparison of images taken far enough on either side of this plane to resolve the grid pattern of the IFU and 
fiber PSFs (i.e. such that the fibers are not overlapping).

To accomplish such measurements, both ends of the cable were mounted on an optical bench, and the slit optics injected with an LED source. To this end, a BlackFly BFLY-U3-05S2M-CS camera was positioned in front of the IFU atop a horizontal translation stage to provide motion along the optical axis. By translating the stage from one side of the common fiber overlap plane, through focus, and to the other, the images in Figure 5 could be taken and analysed for each RHEA@Subaru cable.

Noticeable immediately is that while neither cable maintains a perfectly spaced grid of Airy disks, the original cable demonstrates significantly less well defined fiber core images both before and beyond the common plane, while the fibers in the replacement cable more or less maintain their shape. This can be attributed to a combination of design improvements in the replacement cable, specifically the additional precision enabled by having the near field camera during fabrication, and the more robust design.
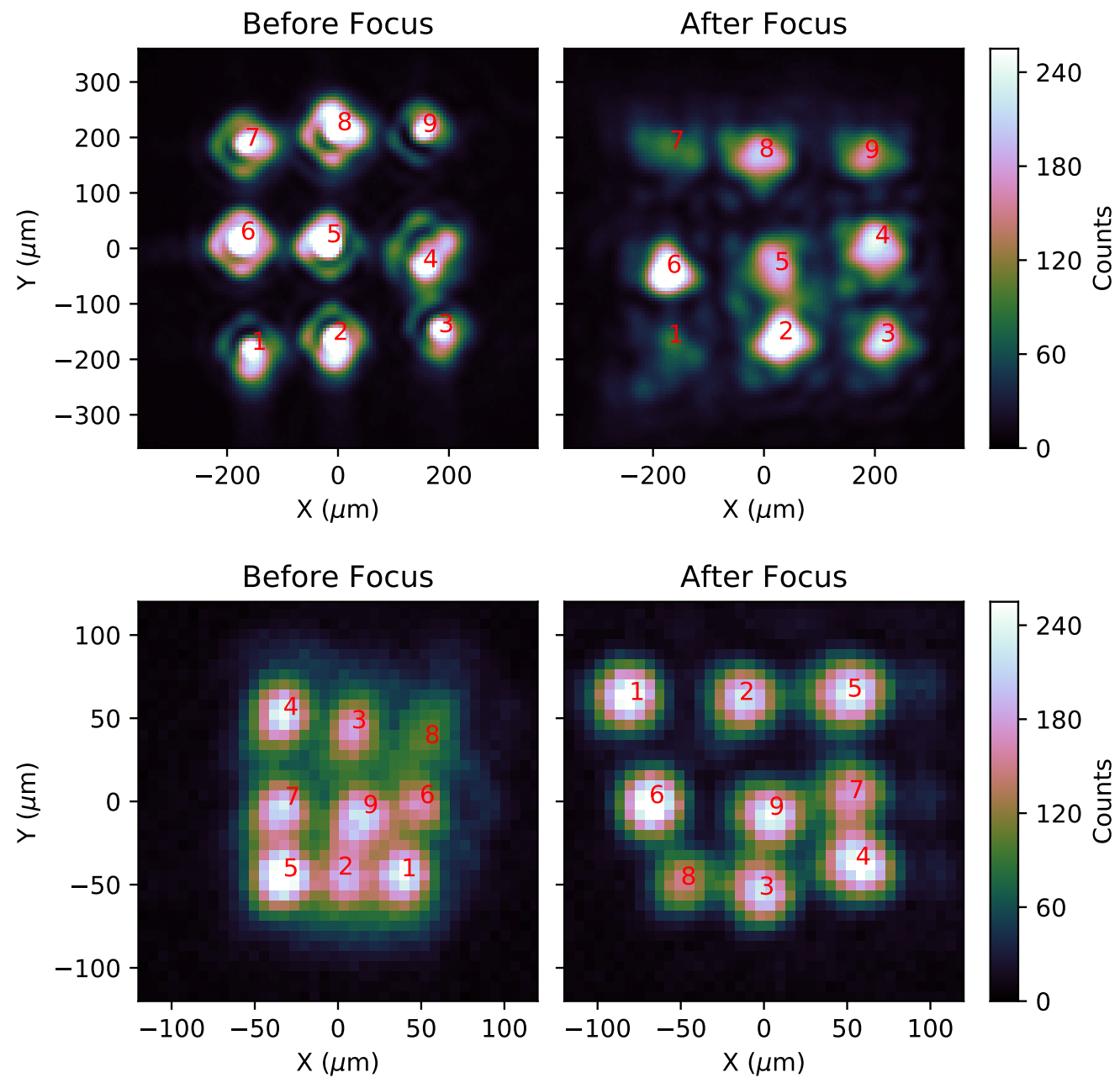

Figure 5. Back-illumination of both RHEA@Subaru cables, with images taken either side of the common fiber source plane. Top Left: original IFU $5 \mathrm{~mm}$ before overlap Top Right: original IFU $5 \mathrm{~mm}$ after overlap. Bottom Left: replacement IFU $2 \mathrm{~mm}$ before overlap Bottom Right: replacement IFU $2 \mathrm{~mm}$ after overlap. Note that some variations in intensity can likely be attributed to uneven illumination at the slit end, as well as the relative alignment quality of each fiber. Each fiber is labeled numerically and will be referenced as such for the remainder of this proceeding.

Figure 6 quantifies what can be seen in Figure 5 by giving numerical $\mathrm{X}$ and $\mathrm{Y}$ misalignment for each fiber relative to an ideally positioned fiber core. These are on average, for the original and replacement cables respectively, misalignments of $(\mathrm{X}=0.85 \pm 0.09 \mu \mathrm{m}, \mathrm{Y}=1.29 \pm 0.09 \mu \mathrm{m})$ and $(\mathrm{X}=0.63 \pm 0.16 \mu \mathrm{m}, \mathrm{Y}=$ 
$0.54 \pm 0.16 \mu \mathrm{m})$ - thus demonstrating a clear improvement in sub-micron positioning accuracy. Considering the original cable, fibers 4,8 , and 7 (within errors) are positioned such that their centers are outside of the ideal fiber core, with the misalignment of at least fiber 4 known since fabrication where it shifted during UV glue curing. Similar incidents of fibers shifting during curing were not observed during the fabrication of the second IFU, likely helped in part due to the tight cavities provided by the glass mask. No fibers from the original cable can be considered aligned with the ideal fiber core, and are instead scattered radially in all X and Y directions. The replacement IFU demonstrates an obvious improvement, with the fibers clustered more tightly around this point, clearly showing the benefits of the cable design and fabrication procedure improvements.
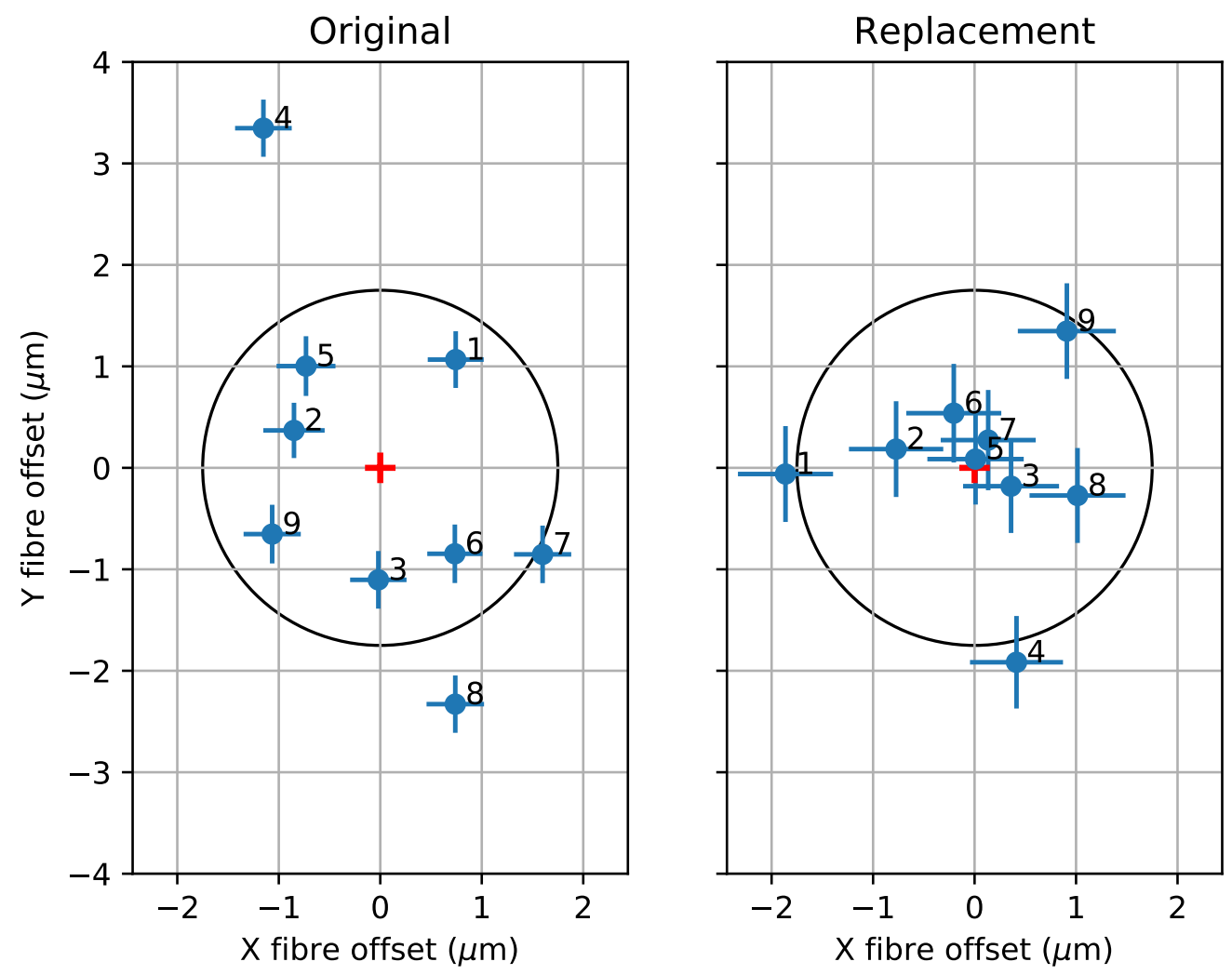

Figure 6. Fiber misalignment in X and Y dimensions (in the plane of the fiber cores) of RHEA@Subaru for Left: original IFU, and Right: replacement IFU. Errors are of \pm 1 px in initial profile center selection using the images in Figure 5 , with the circles representing the core size of an ideally positioned $630 \mathrm{HP}$ SMF $\left(\mathrm{r}_{\text {core }}=1.75 \mu \mathrm{m}\right)$

\section{CABLE THROUGHPUT}

Following characterization of fiber alignment issues, more informed investigation could then be conducted into fiber throughputs. Optimally injecting into RHEA's IFU requires micron-level alignment across multiple axes, a process made easier when it is known which fibers can be expected to perform poorly. Unless otherwise stated, all reported throughputs consist of the following optical setup:

- $7.2 \mathrm{~mm}$ SCExAO input pupil with $31 \%$ secondary obstruction,

- $300 \mathrm{~mm}$ focusing lens (AC254-300-B),

- $1.45 \mathrm{~mm}$ re-focusing lens (354140-B),

- IFU and fiber cable 
- BlackFly BFLY-U3-05S2M-CS camera and lens mounted to image fiber slit (resolving the outputs of each fiber individually),

- Power meter on removable post mounted between pseudo-slit and camera.

To conservatively calculate the predicted maximum throughput, the cables were considered to have $9 \times$ (or $7 \times$ for the original cable) anti-reflection coated interfaces $(99 \%$ each, $96 \%$ each for the $2 \times$ uncoated original surfaces); a beam sampler (96.5\%); $4 \times$ silica-NOA61 glue interfaces, $2 \times 30^{\circ}$ SF11-NOA61 interfaces, and $2 \times$ normal SF11-NOA61 interfaces (97.5\% combined), $12 \mathrm{~m}$ of $630 \mathrm{HP}$ SMF ( $\leq 12 \mathrm{~dB} \mathrm{~km}^{-1}$, or $\left.96 \%\right)$. Fiber coupling was calculated using simulation code written in Python for the optical setup described above, including the designed for pupil-IFU separations of each cable. This resulted in values of $43.9 \%$ and $59.4 \%$ for the original and replacement cables respectively, which the change in throughput primarily due to the different on-sky resolution between the two versions. An additional loss at the second microlens array (96\%) for a fundamental mode was also incorporated, resulting in theoretical maximum throughputs of approximately $33 \%$ for the original cable, and $47 \%$ for it replacement.

With these expectations, and informed by the known fiber offsets, the procedure to observationally measure throughputs was as follows:

1. Inject a laser source into the IFU, coarsely adjusting with reference to the live camera feed of the pseudo-slit end until light emerges from the cable.

2. Determine orientation of now-illuminated pseudo-slit by temporarily switching on calibration source to inject through the simultaneous reference fiber.

3. Finely adjust the optical stages to maximize the light emerging from the best aligned fiber (as per Figure $6)$.

4. Insert the power meter into its mount between the pseudo-slit and camera and continue to finely adjust the stages to optimize the output power. Once maximized, only further $\mathrm{X}$ and $\mathrm{Y}$ shifts will be required to optimally inject into other similarly well-aligned fibers - no additional angular shifts will be necessary.

5. Measure and record the output power for this fiber.

6. Repeat steps 3-5 for each other science fiber in the slit, adjusting only in the $\mathrm{X}$ and $\mathrm{Y}$ dimensions.

7. Mount the power meter before the $300 \mathrm{~mm}$ focus lens and measure the input power.

8. Calculate all throughputs given input and output powers.

Following this procedure, the throughputs in Table 5 were calculated. The replacement cable is again a quantifiable improvement over its predecessor, with all fibers having measurable throughputs and only two having throughputs less than 10\%. Despite this improvement, the cable is worse by a factor of 3 than what theory predicts. Based on observed image quality when the fiber was illuminated in both directions, additional fabrication errors causing mode mismatch seems to be an unlikely cause of this loss. Given the importance of high throughput, we recommend that similar devices are tested at each manufacturing step.

Table 4. Measured throughputs for RHEA's fiber cable. Note that for the original cable, two fibers were so misaligned that their throughputs were unable to be measured per the steps above.

\begin{tabular}{|c|c|c|c|}
\hline \multirow{2}{*}{ Cable } & \multicolumn{2}{|c|}{ Throughput (\%) } & \\
\cline { 2 - 4 } & Theoretical & Measured (per fiber) & $3.9 \times$ \\
\hline Original & 32.7 & $8.0,4.4, \mathbf{8 . 4}, 5 ., 3.2,7.5,7.1$ & $3 \times$ \\
Replacement & 47.1 & $12.8,10.5,4.5,6.8,7.5, \mathbf{1 5 . 8}, 14.3,15.0,11.3$ & \\
\hline
\end{tabular}




\subsection{Chromatism in Alignment Procedure}

In the process of aligning the replacement cable at Subaru by simultaneously injecting the IFU with a red laser source, and back-illuminating with a green laser source, a significant chromatic aberration was observed in the pupil plane. In the absence of this effect, both the incoming red source and back-illuminated green source should overlap. In reality however, the green pupil was offset by several PSF widths from its red counterpart, resulting in poor throughputs for any alignment not taking this into account (i.e. alignment without back-illumination). This heretofore unknown aberration led to the premature assessment of the replacement cable as inferior to the original based on data taken during SCExAO engineering nights, an assessment that motivated much of this work.

No such chromatic aberration was observed in the lab for the original cable, with any effect being of the same order of magnitude as the inherent fiber misalignment. The suspected cause of the effect is the sum of errors introduced when bonding the prisms and microlens array, specifically optical dispersion through thick glue layers, prism rotational-misalignment, and (most-likely) wedges of glue. A wedge of glue between the microlens array and prism of approximately $60 \mu \mathrm{m}$ thick per mm, or $300 \mu \mathrm{m}$ total, could cause this. Although unexpected, this is not inconsistent with lab notes and photos taken during fabrication.

Now that it is known, it can be taken into account during the alignment procedure, mitigating its influence during use. Its existence however does raise further considerations for a final upgrade science cable, or for others fabricating similar optical systems, where efforts have to made to limit the thickness of glue layers, or remove them from the design entirely (i.e. by initially fabricating a single unit of glass to the designed thickness rather than using prisms).

\section{FUTURE WORK}

With RHEA's replacement cable installed and characterized, correcting the modal-noise issue plaguing the original, and improving system throughput, the main remaining task is analysis of preliminary scientific data taken during SCExAO engineering time. The data reduction algorithm to obtain deconvolved spectra and RVs from a resolved target however is nontrivial, and work is ongoing.

\section{SUMMARY}

The RHEA@Subaru module for SCExAO fills a unique niche with its ability to observe at both high spectral and spatial resolutions targets only resolvable with the largest of contemporary telescopes. Following its commissioning, RHEA's original IFU equipped fiber cable was demonstrated to be flawed, being afflicted by modal-noise due to closely packed fibers of similar lengths. A more advanced replacement cable has since been fabricated and installed, correcting the modal noise issue, and increasing system throughput, despite unexpected chromatic aberrations in the pupil plane. Work is ongoing on the complex data reduction pipeline necessary to obtain deconvolved spectra and RVs of the resolved targets able to be observed by RHEA, with data taken during SCExAO engineering nights awaiting reduction.

\section{ACKNOWLEDGMENTS}

This work was partially funded by the Australian Research Council Discovery Project DP120103751. We would like to thank Macquarie Engineering and Technical Services (METS) and the RSAA Manufacturing Workshop for their work in machining parts to complete the spectrograph assembly. Furthermore we thank Dr Benjamin Johnston, Dr Martin Ams and Alex Stokes from OptoFab for their assistance in constructing the fiber cable used for RHEA@Subaru, as well as OptoFab for use of the Nanosecond Laser Micro-Fabrication Facility, and Optical Device Characterisation Suite. ADR acknowledges support from the Australian Government Research Training Program, and the RSAA top up scholarship. We would like to thank the Subaru Telescope and the National Astronomical Observatory of Japan for granting us the opportunity to undertake this project and for their continued assistance throughout. The authors wish to recognize and acknowledge the very significant cultural role and reverence that the summit of Maunakea has always had within the indigenous Hawaiian community. We are most fortunate to have the opportunity to conduct observations from this mountain. 


\section{REFERENCES}

[1] Feger, T., Bacigalupo, C., Bedding, T. R., Bento, J., Coutts, D. W., Ireland, M. J., Parker, Q. A., Rizzuto, A., and Spaleniak, I., "RHEA: the ultra-compact replicable high-resolution exoplanet and Asteroseismology spectrograph," in [SPIE 9147], Ramsay, S. K., McLean, I. S., and Takami, H., eds., Vol. 9147, 91477I, SPIE Proceedings, Montral, Quebec, Canada (July 2014).

[2] Feger, T., Ireland, M. J., Schwab, C., Bento, J., Bacigalupo, C., and Coutts, D. W., "Attaining m s1 level intrinsic Doppler precision with RHEA, a low-cost single-mode spectrograph," Experimental Astronomy , 1-16 (Aug. 2016).

[3] Bento, J., Feger, T., Ireland, M. J., Rains, A., Jovanovic, N., Coutts, D. W., Schwab, C., Arriola, A., and Gross, S., "Performance and future developments of the RHEA single-mode spectrograph," 9908, 99086K99086K-11 (2016).

[4] Jovanovic, N., Martinache, F., Guyon, O., Clergeon, C., Singh, G., Kudo, T., Garrel, V., Newman, K., Doughty, D., Lozi, J., Males, J., Minowa, Y., Hayano, Y., Takato, N., Morino, J., Kuhn, J., Serabyn, E., Norris, B., Tuthill, P., Schworer, G., Stewart, P., Close, L., Huby, E., Perrin, G., Lacour, S., Gauchet, L., Vievard, S., Murakami, N., Oshiyama, F., Baba, N., Matsuo, T., Nishikawa, J., Tamura, M., Lai, O., Marchis, F., Duchene, G., Kotani, T., and Woillez, J., "The Subaru Coronagraphic Extreme Adaptive Optics System: Enabling High-Contrast Imaging on Solar-System Scales," Publications of the Astronomical Society of the Pacific 127, 890-910 (Sept. 2015).

[5] Rains, A. D., Ireland, M. J., Jovanovic, N., Feger, T., Bento, J., Schwab, C., Coutts, D. W., Guyon, O., Arriola, A., and Gross, S., "Precision single mode fibre integral field spectroscopy with the RHEA spectrograph," 9908, 990876-990876-12 (2016). 